This item was submitted to Loughborough's Research Repository by the author.

Items in Figshare are protected by copyright, with all rights reserved, unless otherwise indicated.

\title{
Anti-disturbance fault tolerant initial alignment for inertial navigation system subjected to multiple disturbances
}

PLEASE CITE THE PUBLISHED VERSION

https://doi.org/10.1016/j.ast.2017.10.041

PUBLISHER

(c) Elsevier

VERSION

AM (Accepted Manuscript)

\section{PUBLISHER STATEMENT}

This work is made available according to the conditions of the Creative Commons Attribution-NonCommercialNoDerivatives 4.0 International (CC BY-NC-ND 4.0) licence. Full details of this licence are available at: https://creativecommons.org/licenses/by-nc-nd/4.0/

\section{LICENCE}

CC BY-NC-ND 4.0

\section{REPOSITORY RECORD}

Cao, Songyin, Lei Guo, and Wen-Hua Chen. 2017. "Anti-disturbance Fault Tolerant Initial Alignment for Inertial Navigation System Subjected to Multiple Disturbances”. figshare. https://hdl.handle.net/2134/27526. 


\title{
Anti-disturbance fault tolerant initial alignment for inertial navigation system subjected to multiple disturbances ${ }^{\text {th }}$
}

\author{
Songyin $\mathrm{CaO}^{\mathrm{a}, *}$, Lei Guo ${ }^{\mathrm{b}}$, Wenhua $\mathrm{Chen}^{\mathrm{c}}$ \\ ${ }^{a}$ Department of Automation, College of Information Engineering, Yangzhou University, Yangzhou, 225127, \\ P. R. China. \\ ${ }^{b}$ National Key Laboratory on Aircraft Control Technology, School of Automation and Electronic \\ Engineering, Beihang University, Beijing, 100191, P.R. China. \\ ${ }^{c}$ Department of Aeronautical and Automotive Engineering, Loughborough University, Loughborough, LE11 \\ 3TU, U.K.
}

\begin{abstract}
Modeling error, stochastic error of inertial sensor, measurement noise and environmental disturbance affect the accuracy of an inertial navigation system (INS). In addition, some unpredictable factors, such as system fault, directly affect the reliability of INSs. This paper proposes a new anti-disturbance fault tolerant alignment approach for a class of INSs subjected to multiple disturbances and system faults. Based on modeling and error analysis, stochastic error of inertial sensor, measurement noise, modeling error and environmental disturbance are formulated into different types of disturbances described by a Markov stochastic process, Gaussian noise and a norm-bounded variable, respectively. In order to improve the accuracy and reliability of an INS, an anti-disturbance fault tolerant filter is designed. Then, a mixed dissipative/guarantee cost performance is applied to attenuate the norm-bounded disturbance and to optimize the estimation error. Slack variables and dissipativeness are introduced to reduce the conservatism of the proposed approach. Finally, compared with the unscented Kalman filter (UKF), simulation results for self-alignment of an INS are provided based on experimental data. It can be shown that the proposed method has an enhanced disturbance rejection and attenuation performance with high reliability.
\end{abstract}

Keywords: Inertial Navigation System, Fault Tolerant, Filter, Initial Alignment, Robustness, Multiple Disturbances.

\section{Introduction}

An Inertial Navigation System (INS) is a dead reckoning system that uses gyroscopes, accelerometers and a navigation computer to continuously calculate navigation parameters of

\footnotetext{
This work is partially supported by the National Natural Science Foundation of China (Grant Nos. 61320106010 and 61473249$)$.

* Corresponding author

Email addresses: sycao@yzu.edu.cn (Songyin Cao), lguo@buaa.edu.cn (Lei Guo), W. Chen@lboro.ac.uk (Wenhua Chen) 
a carrier based on Newton's laws of motion (see [1]). INS has been widely applied in various fields. One of the important issue is calibration and alignment, which directly relates to the navigation precision and start-up time of an INS ([2]).

The process of traditional initial alignment includes a coarse alignment stage and a fine alignment stage [3]. Analytic approaches are often applied to the coarse alignment. The most widely used fine alignment method is based on Kalman filter (KF) (see [4, 5]). Kalman filtering, known as linear quadratic estimation (LQE), is an algorithm that uses Bayesian inference and estimates a joint probability distribution over the variables for each time frame. For a nonlinear INS, an adaptive extended Kalman filter (EKF) was proposed to linearize about an estimation of the current mean and covariance for in-flight alignment [6]. In addition, the unscented Kalman filter (UKF) uses the unscented transformation to pick a minimal set of sample points (called sigma points) around the mean for nonlinear systems (see [7] and references therein). Up to now, Kalman filtering type approaches (including $\mathrm{KF}, \mathrm{EKF}$ and UKF) have been successfully applied to initial alignment of INS and obtained satisfactory results. In the filtering problems, not only external disturbances, measurement noises, but also unmodeled dynamics, nonlinear and uncertain dynamics are usually merged into a single disturbance. The accuracy of Kalman filtering type methods could be degraded, since the merged disturbance may not be Gaussian in most INSs. Robust filtering methods aim to achieve robust performance and stability in the presence of bounded modeling errors, and no statistical assumptions on the noises are required $[8,9]$. In $[10,11,12]$, robust filters were proposed to improve the filtering stability and performance for in-flight alignment. Because of the complexity and special characteristics of INS, many other filtering approaches have not been applied to the problem of initial alignment so far.

In most of the above-mentioned design, a single disturbance (either a Gaussian noise or a norm bounded variable) is considered, and Kalman filtering type or robust filtering methods can be used. It has been shown that multiple types of disturbances exist in most practical processes and can be formulated into different mathematical descriptions (see [13]). For example, in a class of INSs, measurement noises, stochastic error of inertial sensor, environmental disturbances and unmodeled dynamics can be modeled by different descriptions [2]. Anti-disturbance control and filtering has been a hot topic in the control field nowadays (see $[13,14]$ and references therein). A linear error model subjected to multiple disturbances was established for an INS in [15], where modeling error and environmental disturbance are merged into a norm bounded variable, measurement noise is assumed to be Gaussian, and inertial sensor error is represented by a first order Gaussian Markov process. Then, a mixed $H_{2} / H_{\infty}$ filtering approach was presented for stationary base self-alignment with enhanced disturbance rejection and attenuation performance. An anti-disturbance initial alignment approach for nonlinear INS was presented in [16], where the nonlinear dynamics and multiple disturbances were respectively analyzed and modelled. The healthy INSs were discussed without sensor failure or performance degradation in $[15,16]$.

Research of fault detection and diagnosis (FDD) as well as fault tolerant control (FTC) has been one of the important aspects in improving the reliability of practical processes (see $[17,18,19,20,21,22,23,24,25,26,27,28]$ and references therein). A velocity-free uncertain attenuation control approach for a class of nonlinear systems with external disturbance 
and multiple actuator faults was addressed in [28]. The change of working temperature and integrated circuit aging could result in the variation of error characteristics or performance degradation for inertial sensors (see [29, 30]). In [29], the dynamic thermal error induced by accelerations of a micro-electro-mechanical system (MEMS) gyroscope is analyzed based on the thermal interferential moment. Additionally, the change of working environment can lead to the variation of the noise covariance [6]. Therefore, some unpredictable factors will affect the reliability of navigation system directly. In [31], failure modes and models of integrated INS/GPS systems were systematically classified based on their effect on the integration process and on each individual component of the integrated system. In [32], a fault detection methodology was proposed for both low frequency faults in an inertial measurement unit (IMU) caused by bias in the sensor readings and the misalignment of the unit, and high frequency faults from the GPS receiver caused by multi-path errors for the integrated IMU/GPS navigation system. A fault-detection algorithm based on the sequential probability ratio test and Chi-Square Test was proposed for a redundant multisensor navigation system for hypersonic cruise vehicles in [33]. In [34], an adaptive two-stage EKF was introduced to improve the reliability of coupled INS/GPS system with unknown bias fault. A magnetic compass fault detection approach for GPS/INS/Magnetic compass integrated navigation systems was proposed in [35], where the abrupt fault was supposed to be caused by the hard iron and soft iron effect. In [36], a fault tolerant Strapdown INS (SINS)/Global Navigation Satellite System (GNSS) approach was presented based on modified EKF and divided difference KF. However, to the best of our knowledge, the problems of fault tolerant alignment are still under research, which motivate us to study this meaningful and challenging topic.

In this paper, an anti-disturbance fault tolerant alignment approach is proposed to improve the accuracy and reliability of an INS. Firstly, a new INS error model subjected to multiple disturbances and system fault is established instead of a healthy INS with a single disturbance. The multiple disturbances are analyzed and modeled respectively. The stochastic error of the inertial sensor is described by a first order Gaussian Markov process. The modeling uncertainties, environmental disturbances and non-Gaussian noises are merged into a norm bounded variable. Then, a fault tolerant filter is proposed with disturbance rejection and attenuation performance. In the proposed scheme, compensation terms are designed to compensate the stochastic error of inertial sensor and to accommodate the sensor fault. Dissipative performance is adopted to attenuate the norm bounded uncertain disturbances and the guarantee cost index is applied to optimize the estimation error respectively. Different from robust $H_{\infty}$ and passivity approaches, the proposed approach has less conservatism. Finally, with collected experimental data, simulations and comparisons with unscented Kalman filter (UKF) are carried out for stationary base alignment of an INS. It is shown that the proposed approach has higher accuracy and reliability. The remainder of this paper is organized as follows. In Section II, a new INS error model subjected to multiple disturbances and sensor fault for stationary base is established. In Section III, an anti-disturbance fault tolerant filter is proposed for the initial alignment problem. Simulations based on experimental data are given in Section IV to show the efficiency of the proposed method. Conclusions are given in Section V. 


\section{Problem Formulation and Preliminaries}

In this paper, the local level $N E D$ (North-East-Down) frame is selected as the navigation frame. For INS on a stationary base, position and velocity errors can be ignored. Therefore, a modified large azimuth misalignment angle error model of SINS can be formulated as follows (see [37])

$$
\dot{\phi}=\left(I-C_{n}^{p}\right) w_{i e}^{n}+C_{b}^{n} \varepsilon^{b}
$$

where $\phi=\left[\begin{array}{lll}\phi_{N}(t) & \phi_{E}(t) & \phi_{D}(t)\end{array}\right]^{T}$ are three misalignment angles, three gyroscope drifts are $\varepsilon^{b}=\left[\begin{array}{lll}\varepsilon_{x}(t) & \varepsilon_{y}(t) & \varepsilon_{z}(t)\end{array}\right]^{T}$. I is a unit matrix with proper dimension. The subscripts $N, E$ and $D$ are corresponding to north, east and down of the navigation frame $n$, and the subscripts $x, y$ and $z$ denote front, right, down of the body frame $b$ respectively. In many practical processes, heading error is much larger than leveling errors, so that we take no account of the linearization of azimuth misalignment angle for the sake of relatively large modeling error. $C_{n}^{p}$ represents the transformation matrix between the navigation frame $n$ and computation frame $p$, which can be further described by

$$
C_{n}^{p}=\left[\begin{array}{ccc}
\cos \phi_{D}(t) & \sin \phi_{D}(t) & -\phi_{E}(t) \\
-\sin \phi_{D}(t) & \cos \phi_{D}(t) & \phi_{N}(t) \\
\bar{\phi}_{E}(t) & \bar{\phi}_{N}(t) & 1
\end{array}\right]
$$

where

$$
\bar{\phi}_{E}(t)=\phi_{N}(t) \sin \phi_{D}(t)+\phi_{E}(t) \cos \phi_{D}(t)
$$

and

$$
\bar{\phi}_{N}(t)=\phi_{E}(t) \sin \phi_{D}(t)-\phi_{N}(t) \cos \phi_{D}(t)
$$

$w_{i e}^{n}=\left[\begin{array}{lll}\Omega_{N} & 0 & -\Omega_{D}\end{array}\right]^{T}$ represents the earth rate in frame $n$. The superscript $n$ denote the navigation frame. $\Omega_{D}$ and $\Omega_{N}$ are the down and north components of earth rate, respectively. $C_{b}^{n}$ is the attitude matrix relating the body frame $b$ with navigation frame $n$, which can be computed by the roll, pitch and yaw angles of the carrier, denoted as

$$
C_{b}^{n}=\left\{C_{i j}\right\}_{i, j=1,2,3}
$$

Because of the variations of working temperature and aging of integrated circuit, there may exist unpredictable performance degradation or fault including bias in the sensor readings and the misalignment of the unit (see [32, 29, 31]). Modeling error, internal sensor noises and environmental disturbances are merged into a norm bounded disturbance in the SINS. Thus, the error equation subjected to multiple disturbances and sensor faults can be further described as follows

$$
\left[\begin{array}{c}
\dot{\phi}_{N}(t) \\
\dot{\phi}_{E}(t) \\
\dot{\phi}_{D}(t)
\end{array}\right]=A_{0}\left[\begin{array}{l}
\phi_{N}(t) \\
\phi_{E}(t) \\
\phi_{D}(t)
\end{array}\right]+C_{b}^{n}\left[\begin{array}{c}
\varepsilon_{x}(t) \\
\varepsilon_{y}(t) \\
\varepsilon_{z}(t)
\end{array}\right]+B_{2} C_{b}^{n} \omega_{f 0}(t)+g_{0}(\phi)+B_{1} d(t)
$$


where the matrix $A_{0}$ is defined as

$$
A_{0}=\left[\begin{array}{ccc}
0 & -\Omega_{D} & 0 \\
\Omega_{D} & 0 & 0 \\
0 & 0 & 0
\end{array}\right]
$$

and nonlinear function is

$$
g_{0}(\phi)=\left[\begin{array}{lll}
\Omega_{N}\left(1-\cos \phi_{D}\right) & \Omega_{N} \sin \phi_{D} & -\Omega_{N} \bar{\phi}_{E}
\end{array}\right]^{T}
$$

$\omega_{f 0}(t)$ is the sensor faults for the concerned SINS. $d(t)$ is the norm bounded disturbance. $B_{1}$ and $B_{2}$ are two coefficient matrices of the system with suitable dimensions.

The principle of self-alignment for SINS is to measure the earth rate $w_{i e}$ and the local gravity acceleration $g$ by the gyroscopes and accelerometers, respectively. Similarly to [1, $15,16]$, the level accelerometer outputs $f_{E}(t), f_{N}(t)$ and east gyro output $w_{E}(t)$ are selected as the measured signals. Then, the measurement output equation is selected as follows

$$
\left[\begin{array}{c}
f_{N}(t) \\
f_{E}(t) \\
w_{E}(t)
\end{array}\right]=C_{0}\left[\begin{array}{l}
\phi_{N}(t) \\
\phi_{E}(t) \\
\phi_{D}(t)
\end{array}\right]+D_{0} \omega_{0}(t)+D_{0} \omega_{f 0}(t)+h_{0}(\phi)+D_{1} d(t)
$$

where

$$
\begin{gathered}
C_{0}=\left[\begin{array}{ccc}
0 & g & 0 \\
-g & 0 & 0 \\
\Omega_{D} & 0 & 0
\end{array}\right] \\
D_{0}=\left[\begin{array}{ccccc}
0 & 0 & 0 & C_{11} & C_{12} \\
0 & 0 & 0 & C_{21} & C_{22} \\
C_{11} & C_{12} & C_{13} & 0 & 0
\end{array}\right]
\end{gathered}
$$

and nonlinear function

$$
h_{0}(\phi)=\left[\begin{array}{lll}
0 & 0 & \Omega_{N} \sin \phi_{D}
\end{array}\right]^{T}
$$

In (7), the inertial sensor errors are denoted by

$$
\omega_{0}(t)=\left[\begin{array}{lllll}
\varepsilon_{x}(t) & \varepsilon_{y}(t) & \varepsilon_{z}(t) & \nabla_{x}(t) & \nabla_{y}(t)
\end{array}\right]^{T}
$$

where $\nabla$ represents the accelerometer measurement error. $D_{1}$ is a coefficient matrix of the system with suitable dimensions.

Similar to $[15,16]$, based on the relationship between navigation frame and body frame, the equations (4) and (7) can be further formulated as follows

$$
\left\{\begin{array}{l}
{\left[\begin{array}{c}
\dot{\phi}_{N}(t) \\
\dot{\phi}_{E}(t) \\
\dot{\phi}_{D}(t)
\end{array}\right]=A_{0}\left[\begin{array}{l}
\phi_{N}(t) \\
\phi_{E}(t) \\
\phi_{D}(t)
\end{array}\right]+\left[\begin{array}{l}
\varepsilon_{N}(t) \\
\varepsilon_{E}(t) \\
\varepsilon_{D}(t)
\end{array}\right]+g_{0}(\phi)+B_{1} d(t)+B_{2} \omega_{f}(t)} \\
{\left[\begin{array}{c}
f_{N}(t) \\
f_{E}(t) \\
w_{E}(t)
\end{array}\right]=C_{0}\left[\begin{array}{c}
\phi_{N}(t) \\
\phi_{E}(t) \\
\phi_{D}(t)
\end{array}\right]+\left[\begin{array}{c}
\nabla_{N}(t) \\
\nabla_{E}(t) \\
\varepsilon_{E}(t)
\end{array}\right]+h_{0}(\phi)+D_{1} d(t)+D_{2} \omega_{f}(t)}
\end{array}\right.
$$


The Taylor series expansions of sinusoidal and cosine functions can be denoted as follows

$$
\sin (\theta)=\theta-\frac{\theta^{3}}{6}+\frac{\theta^{5}}{120}+o\left(\theta^{5}\right)
$$

and

$$
\cos (\theta)=1-\frac{\theta^{2}}{2}+\frac{\theta^{4}}{24}+o\left(\theta^{5}\right)
$$

$\theta$ is supposed to be in the neighborhood interior of zero, then $o\left(\theta^{5}\right)$ is the high order infinitesimal term. Applying (12) and (13) to the nonlinear terms of the error model (11), the nonlinear INS error system (11) can be formulated by

$$
\left\{\begin{array}{l}
\dot{x}(t)=A x(t)+G g(x(t))+B\left[\omega(t)+\omega_{f}(t)\right]+B_{1} d(t) \\
y(t)=C x(t)+H h(x(t))+D\left[\omega(t)+\omega_{f}(t)\right]+D_{1} d(t)
\end{array}\right.
$$

where the system state

$$
x^{T}(t)=\left[\begin{array}{lll}
\phi_{N}(t) & \phi_{E}(t) & \phi_{D}(t)
\end{array}\right]
$$

the system output variable is

$$
y^{T}(t)=\left[\begin{array}{lll}
f_{N}(t) & f_{E}(t) & w_{E}(t)
\end{array}\right]
$$

and $\omega_{f}$ is the sensor fault in navigation frame. The inertial sensor errors in frame $n$ are

$$
\omega(t)=\left[\begin{array}{lllll}
\varepsilon_{N}(t) & \varepsilon_{E}(t) & \varepsilon_{D}(t) & \nabla_{N}(t) & \nabla_{E}(t)
\end{array}\right]^{T}
$$

Similar to $[15,16,38]$, stochastic error of inertial sensor is supposed to be a first-order Gaussian Markov process, which can be described by

$$
\dot{\omega}(t)=W \omega(t)+E \delta(t)
$$

where $W=\operatorname{diag}\left\{-\frac{1}{\tau_{1}}, \cdots,-\frac{1}{\tau_{5}}\right\}$ and $\tau_{i}(i=1, \cdots, 5)$ are correlation times of Markov process. $E$ represents the coefficient matrix and can be determined by the properties of the selected inertial sensors. $\delta(t)$ is the Gaussian noise. $B_{1}$ and $D_{1}$ are denoted in (4) and (7), respectively. The coefficient matrices in the nonlinear model (14) are determined by the kinetic and kinematic equations of INS, and denoted as

$$
\begin{gathered}
A=\left[\begin{array}{ccc}
0 & -\Omega_{D} & 0 \\
\Omega_{D} & 0 & \Omega_{N} \\
0 & -\Omega_{N} & 0
\end{array}\right], C=\left[\begin{array}{ccc}
0 & g & 0 \\
-g & 0 & 0 \\
\Omega_{D} & 0 & \Omega_{N}
\end{array}\right], \\
B=\left[\begin{array}{lllll}
1 & 0 & 0 & 0 & 0 \\
0 & 1 & 0 & 0 & 0 \\
0 & 0 & 1 & 0 & 0
\end{array}\right], D=\left[\begin{array}{lllll}
0 & 0 & 0 & 1 & 0 \\
0 & 0 & 0 & 0 & 1 \\
0 & 1 & 0 & 0 & 0
\end{array}\right] .
\end{gathered}
$$


The nonlinear terms can be denoted as

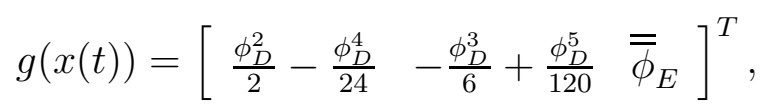

and

$$
h(x(t))=\left[\begin{array}{lll}
0 & 0 & -\frac{\phi_{D}^{3}}{6}+\frac{\phi_{D}^{5}}{120}
\end{array}\right]^{T}
$$

where

$$
\overline{\bar{\phi}}_{E}=\phi_{N}\left(\phi_{D}-\frac{\phi_{D}^{3}}{6}+\frac{\phi_{D}^{5}}{120}\right)+\phi_{E}\left(-\frac{\phi_{D}^{2}}{2}+\frac{\phi_{D}^{4}}{24}\right)
$$

For the stationary base initial alignment, the initial misalignment angles should be in a proper interval. Therefore, the high order infinitesimal term $o\left(\phi_{D}^{5}\right)$ can be merged into the norm bounded disturbance $d(t)$. Correspondingly, the parameter matrices are denoted by

$$
G=\left[\begin{array}{ccc}
\Omega_{N} & 0 & 0 \\
0 & \Omega_{N} & 0 \\
0 & 0 & -\Omega_{N}
\end{array}\right], H=\left[\begin{array}{ccc}
0 & 0 & 0 \\
0 & 0 & 0 \\
0 & 0 & \Omega_{N}
\end{array}\right]
$$

It is noted that $g(0)=0$ and $h(0)=0$ hold. The nonlinear vector function $g(x(t))$ is supposed to satisfy

$$
\left\|g\left(x_{1}(t)\right)-g\left(x_{2}(t)\right)\right\| \leq\left\|U_{1}\left(x_{1}(t)-x_{2}(t)\right)\right\|
$$

for any $x_{1}(t)$ and $x_{2}(t)$, and for a known matrix $U_{1}$. Similarly, the nonlinear vector function $h(x(t))$ satisfies

$$
\left\|h\left(x_{1}(t)\right)-h\left(x_{2}(t)\right)\right\| \leq\left\|U_{2}\left(x_{1}(t)-x_{2}(t)\right)\right\|
$$

for any $x_{1}(t)$ and $x_{2}(t)$, and for a known matrix $U_{2}$. After the process of coarse alignment on a stationary base, the misalignment angles are quite small so are supposed to satisfy $|\phi| \leq \pi / 18$ in this paper. Then, the constant matrices $U_{1}$ and $U_{2}$ are selected as

$$
U_{1}=\left[\begin{array}{ccc}
0 & 0 & \frac{\pi}{18} \\
0 & 0 & \frac{\pi^{2}}{648} \\
\frac{\pi}{18} & \frac{\pi^{2}}{648} & \frac{18 \pi+\pi^{2}}{324}
\end{array}\right], U_{2}=\left[\begin{array}{ccc}
0 & 0 & 0 \\
0 & 0 & 0 \\
0 & 0 & \frac{\pi^{2}}{648}
\end{array}\right]
$$

Remark 1. In most previous results, such as [5, 6, 37], stochastic error of inertial sensor is simplified to be a random constant. In this article, we adopt a first-order Markov process (15) to represent the stochastic error of inertial sensor, which can approximate the physical random processes with improved accuracy. In addition, the modeling error and environmental disturbance of SINS are merged into the norm bounded disturbance $d(t)$ rather than a Gaussian noise. Different from [5, 6, 15, 16], performance degradation or faults of the inertial sensors are further discussed to improve the reliability of INS in this article.

In this section, a new nonlinear SINS model subjected to multiple disturbances and sensor faults is established. Then, the initial alignment problem is transformed into a fault tolerant filter design problem for a class of nonlinear systems with multiple disturbances and faults. 


\section{Anti-Disturbance Fault Tolerant Filter Design}

This section is to design an anti-disturbance fault tolerant filter for estimating misalignment angles for the alignment problem described in Section II. In the following, we will analyze the characteristics of various disturbances, and respectively describe the different types of disturbances. In order to improve the accuracy, both disturbance rejection and attenuation performances should be achieved simultaneously. Different from Kalmam type filters, compensation terms will be designed to reject inertial sensor errors and to accommodate sensor faults in the proposed filter. For this purpose, a mixed multi-objective filter is constructed as follows

$$
\left\{\begin{aligned}
\dot{\widehat{x}}(t) & =A \widehat{x}(t)+G g(\widehat{x}(t))+L[y(t)-\widehat{y}(t)]+u_{c 1}(t) \\
\widehat{y}(t) & =C \widehat{x}(t)+H h(\widehat{x}(t))+u_{c 2}(t) \\
\dot{\widehat{\omega}}(t) & =W \widehat{\omega}(t)+K_{1}[y(t)-\widehat{y}(t)] \\
\dot{\hat{\omega}}_{f}(t) & =K_{2}[y(t)-\widehat{y}(t)]
\end{aligned}\right.
$$

where $\widehat{x}(t)$ is the estimation of state $x(t), \widehat{y}(t)$ represents the estimation of output signal $y(t)$. $\widehat{\omega}(t)$ is the estimation of inertial sensor errors $\omega(t) \cdot \hat{\omega}_{f}(t)$ is an adaptive estimation term, which is adopted to estimate the sensor faults. We use $u_{c 1}(t)=B \widehat{\omega}(t)+B \hat{\omega}_{f}(t)$ and $u_{c 2}(t)=D \widehat{\omega}(t)+D \hat{\omega}_{f}(t)$ as the compensation terms, which are applied to reject inertial sensor errors and accommodate sensor faults. $K_{1}, K_{2}$ and $L$ are filter gains to be determined later.

Denoting $\widetilde{x}(t)=x(t)-\widehat{x}(t), \widetilde{\omega}(t)=\omega(t)-\widehat{\omega}(t), \widetilde{\omega}_{f}(t)=\omega_{f}(t)-\hat{\omega}_{f}(t), \widetilde{f}(t)=f(x(t))-$ $f(\widehat{x}(t))$ and $\widetilde{g}(t)=g(x(t))-g(\widehat{x}(t))$, the estimation error system yields

$$
\left\{\begin{aligned}
\dot{\tilde{x}}(t)= & (A-L C) \widetilde{x}(t)+G \widetilde{g}((t))-L H \widetilde{h}(t) \\
& +(B-L D) \widetilde{\omega}(t)+\left(B_{1}-L D_{1}\right) d(t)+(B-L D) \widetilde{\omega}_{f}(t) \\
\dot{\tilde{\omega}}(t)= & \left(W-K_{1} D\right) \widetilde{\omega}(t)-K_{1} C \widetilde{x}(t)-K_{1} H \widetilde{h}(t) \\
& -K_{1} D \widetilde{\omega}_{f}(t)+E \delta(t)-K_{1} D_{1} d(t) \\
\dot{\tilde{\omega}}_{f}(t)= & \dot{\omega}_{f}(t)-K_{2} D \widetilde{\omega}(t)-K_{2} C \widetilde{x}(t)-K_{2} \widetilde{\omega}(t) \\
& -K_{2} D_{1} d(t)-K_{2} H \widetilde{h}(t)
\end{aligned}\right.
$$

After the inertial sensor errors and faults are compensated by their estimations, the next step is to attenuate the norm bounded disturbances $d(t)$ and $\dot{\omega}_{f}(t)$. We select the dissipative performance to attenuate the norm bounded disturbances, and guarantee cost index to optimize the estimation error. Combining the dissipative and guarantee cost reference outputs with estimation error Equation (22) yields

$$
\left\{\begin{aligned}
\dot{X}(t)= & (\bar{A}-\bar{L} \bar{C}) X(t)+\bar{G} g(X(t))-\bar{L} H h(X(t)) \\
& +\left(\bar{B}_{1}-\bar{L} \bar{D}_{1}\right) \bar{d}(t)+\bar{E} \delta(t) \\
z_{d}(t)= & \bar{C}_{d} X(t) \\
z_{2}(t)= & \bar{C}_{2} X(t)
\end{aligned}\right.
$$


where

$$
\begin{gathered}
X(t)=\left[\begin{array}{c}
\widetilde{x}(t) \\
\widetilde{\omega}(t) \\
\widetilde{\omega}_{f}(t)
\end{array}\right], \bar{A}=\left[\begin{array}{ccc}
A & B & B \\
0 & W & 0 \\
0 & 0 & 0
\end{array}\right], \bar{L}=\left[\begin{array}{c}
L \\
K_{1} \\
K_{2}
\end{array}\right], \\
\bar{G}=\left[\begin{array}{c}
G \\
0 \\
0
\end{array}\right], \bar{B}_{1}=\left[\begin{array}{cc}
B_{1} & 0 \\
0 & 0 \\
0 & I
\end{array}\right], \bar{E}=\left[\begin{array}{c}
0 \\
E \\
0
\end{array}\right], \bar{d}(t)=\left[\begin{array}{c}
d(t) \\
\dot{\omega}_{f}(t)
\end{array}\right], \\
\bar{C}=\left[\begin{array}{ccc}
C & D & D
\end{array}\right], \bar{D}_{1}=\left[\begin{array}{ll}
D_{1} & 0
\end{array}\right], \\
g(X(t))=\widetilde{g}(t), h(X(t))=\widetilde{h}(t), \\
\bar{C}_{2}=\left[\begin{array}{lll}
C_{21} & C_{22} & C_{23}
\end{array}\right], \bar{C}_{d}=\left[\begin{array}{lll}
C_{d 1} & C_{d 2} & C_{d 3}
\end{array}\right] .
\end{gathered}
$$

$z_{d}(t), z_{2}(t)$ are dissipative and guarantee cost performance outputs respectively. $C_{21}, C_{22}$, $C_{23}, C_{d 1}, C_{d 2}$ and $C_{d 3}$ are selected weighting matrices.

After the stochastic error and bias fault of inertial sensor are rejected in the proposed filter, we will use the mixed dissipative/guaranteed cost performance to attenuate the norm bounded disturbance and the Gaussian noise. Sone definitions will be introduced in the following.

Definition 1. For a real constant $\tau$ and any given vectors $x(t), y(t)$, the inner product operator of vectors is defined as

$$
(x(t), y(t))_{\tau}=\int_{0}^{\tau} x^{T}(t) y(t) d t
$$

Before proceeding further, we consider a general linear system

$$
\left\{\begin{array}{l}
\dot{\xi}(t)=\overline{\bar{A}} \xi(t)+\overline{\bar{B}} \eta(t) \\
z(t)=\overline{\bar{C}} \xi(t)
\end{array}\right.
$$

where $\xi(t)$ is the state variable, $\eta(k)$ is the norm bounded disturbance input and $z(t)$ is the reference output. $\overline{\bar{A}}, \overline{\bar{B}}$ and $\overline{\bar{C}}$ represent the coefficient matrices with suitable dimensions.

Definition 2. For any $\tau>0$ and norm bounded disturbance $\eta(t)$, if there exists constant $\gamma>0$ satisfying the following inequality

$$
(z(t), Q z(t))_{\tau}+2(z(t), S \eta(t))_{\tau}+(\eta(t), R \eta(t))_{\tau}>\gamma(\eta(t), \eta(t))_{\tau}
$$

where $Q, R$ are symmetric matrices and $S$ is a matrix with suitable dimensions, then the concerned system $(25)$ is strictly $(Q, S, R)$ dissipative. 
Definition 3. The guarantee cost performance index for (23) is defined as $J_{2}=\left\|z_{2}(t)\right\|^{2}$.

Definition 4. The dissipative performance index for (23) is defined as

$$
J(\tau, \gamma)=\left(z_{d}(t), Q z_{d}(t)\right)_{\tau}+2\left(z_{d}(t), S \bar{d}(t)\right)_{\tau}+(\bar{d}(t), R-\gamma I \bar{d}(t))_{\tau}
$$

At this stage, the objective is to find $\bar{L}$ such that system $(23)$ is strictly $(Q, S, R)$ dissipative and satisfies the mixed performance index.

Theorem 1. For the constants $\lambda_{1}>0, \lambda_{2}>0$, tuning parameters $\alpha>0$ and matrices $\bar{C}_{2}$, $\bar{C}_{d}, Q, S$ and $R$, if the following LMI-based optimization problem:

$$
\min \left\{X^{T}(0) P_{1} X(0)\right\}
$$

subject to

$$
\left[\begin{array}{ccccccc}
\Xi_{1} & \Xi_{12} & P_{2} \bar{G} & -R_{1} H & \Xi_{15} & \bar{C}_{d}^{T} Q_{-} & \bar{C}_{2}^{T} \\
* & \Xi_{2} & \alpha P_{2} \bar{G} & -\alpha R_{1} H & \Xi_{25} & 0 & 0 \\
* & * & -\lambda_{1} I & 0 & 0 & 0 & 0 \\
* & * & * & -\lambda_{2} I & 0 & 0 & 0 \\
* & * & * & * & \gamma I-R & 0 & 0 \\
* & * & * & * & * & -I & 0 \\
* & * & * & * & * & * & -I
\end{array}\right]<0
$$

where $Q_{-}$satisfies $-Q=Q_{-}^{T} Q_{-}$, and

$$
\begin{gathered}
\Xi_{1}=\operatorname{sym}\left(P_{1} \bar{A}-R_{1} \bar{C}\right)+\lambda_{1} U_{1}^{T} U_{1}+\lambda_{2} U_{2}^{T} U_{2}, \\
\Xi_{12}=P_{1}-P_{2}+\alpha\left(P_{2} \bar{A}-R_{1} \bar{C}\right)^{T}, \\
\Xi_{15}=P_{2} \bar{B}_{1}-R_{1} \bar{D}_{1}-\bar{C}_{d}^{T} S, \Xi_{2}=-\alpha P_{2}-\alpha P_{2}^{T}, \\
\Xi_{25}=\alpha\left(P_{2} \bar{B}_{1}-R_{1} \bar{D}_{1}\right) .
\end{gathered}
$$

are feasible with respect to constant $\gamma>0$, matrices $P_{1}>0, P_{2}$ and $R_{1}$, then there exists a mixed multi-objective filter with gains $\bar{L}=P_{2}^{-1} R_{1}$ such that the error system (23) is strict $(Q, S, R)$ dissipative and satisfies $J_{2} \leq X^{T}(0) P_{1} X(0)$ and $J(\tau, \gamma)>0$.

A real symmetric matrix $M>(\geq) 0$ denotes $M$ being a positive definite (positive semidefinite) matrix, and $M>(\geq) N$ means $M-N>(\geq) 0$. The symmetric terms in a symmetric matrix are denoted by $*$. The symbol $\operatorname{sym}()$ represents $\operatorname{sym}(\Theta):=\Theta+\Theta^{T}$. 
Proof. Define the Lyapunov function candidate as follows

$$
\begin{aligned}
V(t)= & X^{T}(t) P_{1} X(t)+\lambda_{1} \int_{0}^{t}\left[\left\|U_{1} X(\tau)\right\|^{2}-\|g(X(\tau))\|^{2}\right] d \tau \\
& +\lambda_{2} \int_{0}^{t}\left[\left\|U_{2} X(\tau)\right\|^{2}-\|h(X(\tau))\|^{2}\right] d \tau
\end{aligned}
$$

It is verified that $V(t) \geq 0$ holds for all arguments. Variable $t$ will be omitted in the following procedures for the sake of simplicity, if it does not cause any confusion. Indeed, the Lyapunov function $V(t)$ can also be denoted as follows

$$
\begin{aligned}
V= & {\left[\begin{array}{c}
X \\
\dot{X}
\end{array}\right]^{T}\left[\begin{array}{cc}
I & 0 \\
0 & 0
\end{array}\right]\left[\begin{array}{cc}
P_{1} & 0 \\
P_{2}^{T} & \alpha P_{2}^{T}
\end{array}\right]\left[\begin{array}{c}
X \\
\dot{X}
\end{array}\right]+\lambda_{1} \int_{0}^{t}\left[\left\|U_{1} X(\tau)\right\|^{2}-\|g(X(\tau))\|^{2}\right] d \tau } \\
& +\lambda_{2} \int_{0}^{t}\left[\left\|U_{2} X(\tau)\right\|^{2}-\|h(X(\tau))\|^{2}\right] d \tau
\end{aligned}
$$

Along with the trajectories of system (23), it can be shown that

$$
\begin{aligned}
\dot{V}= & 2 X^{T} P_{1} \dot{X}+\lambda_{1}\left[\left\|U_{1} X\right\|^{2}-\|g(X)\|^{2}\right]+\lambda_{2}\left[\left\|U_{2} X\right\|^{2}-\|h(X)\|^{2}\right] \\
= & 2\left[\begin{array}{c}
X \\
\dot{X}
\end{array}\right]^{T}\left[\begin{array}{cc}
P_{1} & P_{2} \\
0 & \alpha P_{2}
\end{array}\right]\left[\begin{array}{c}
\dot{X} \\
0
\end{array}\right]+\lambda_{1}\left[\left\|U_{1} X\right\|^{2}-\|g(X)\|^{2}\right] \\
& +\lambda_{2}\left[\left\|U_{2} X\right\|^{2}-\|h(X)\|^{2}\right] \\
= & 2 X^{T} P_{1} \dot{X}-2 X^{T} P_{2} \dot{X}+2 X^{T} P_{2}(\bar{A}-\bar{L} \bar{C}) X+2 \dot{X}^{T} \alpha P_{2}(\bar{A}-\bar{L} \bar{C}) X \\
& -2 \dot{X}^{T} \alpha P_{2} \dot{X}+2 X^{T} P_{2}\left(\bar{B}_{1}-\bar{L} \bar{D}_{1}\right) \bar{d}+2 X^{T} P_{2} \bar{G} g(X) \\
& +2 \dot{X}^{T} \alpha P_{2}\left(\bar{B}_{1}-\bar{L}_{1}\right) \bar{d}+2 \dot{X}^{T} \alpha P_{2} \bar{G} g(X) \\
& +\lambda_{1} X^{T} U_{1}^{T} U_{1} X+\lambda_{2} X^{T} U_{2}^{T} U_{2} X-2 X^{T} P_{2} \bar{L} H h(X) \\
& -2 \dot{X}^{T} \alpha P_{2} \bar{L} H h(X)-\lambda_{1}\|g(X)\|^{2}-\lambda_{2}\|h(X)\|^{2}
\end{aligned}
$$

In the absence of $\bar{d}(t)$ (i. e. $\bar{d}(t)=0)$, it can be concluded that

$$
\dot{V}(t)=s^{T}(t)\left[\Phi-\zeta(t) \zeta^{T}(t)\right] s(t)
$$

where

$$
\begin{gathered}
\Phi=\left[\begin{array}{cccc}
\Xi_{1}+\bar{C}_{2}^{T} \bar{C}_{2} & \Xi_{12} & P_{2} \bar{G} & -R_{1} H \\
* & \Xi_{2} & \alpha P_{2} \bar{G} & -\alpha R_{1} H \\
* & * & -\lambda_{1} I & 0 \\
* & * & * & -\lambda_{2} I
\end{array}\right] \\
s^{T}(t)=\left[\begin{array}{llll}
X^{T} & \dot{X}^{T} & g^{T}(X) & h^{T}(X)
\end{array}\right]
\end{gathered}
$$




$$
\zeta^{T}(t)=\left[\bar{C}_{2} \quad 0 \quad 0 \quad 0\right]
$$

It can be seen by using of Schur complement formula that (29) leads to $\Phi<0$. When $\Phi<0$ holds, we have

$$
\dot{V}(t)<-s^{T}(t) \zeta(t) \zeta^{T}(t) s(t)=-z_{2}^{T} z_{2} \leq 0
$$

Consider the auxiliary function as follows

$$
J_{0}=z_{2}^{T}(t) z_{2}(t)+\dot{V}(t)
$$

It can be verified that $J_{0} \leq s^{T}(t) \Phi s(t)$ holds.

In the presence of $d_{0}(t)$, it can be concluded that

$$
V(\tau)-J(\tau, \gamma)=\int_{0}^{\tau} s_{0}^{T}(t) \Psi s_{0}(t)
$$

where $s_{0}^{T}(t)=\left[\begin{array}{ll}s^{T}(t) & d_{0}^{T}(t)\end{array}\right]$, and

$$
\Psi=\left[\begin{array}{ccccc}
\Xi_{1}-\bar{C}_{d}^{T} Q \bar{C}_{d} & \Xi_{12} & P_{2} \bar{G} & -R_{1} H & \Xi_{15} \\
* & \Xi_{2} & \alpha P_{2} \bar{G} & -\alpha R_{1} H & \Xi_{25} \\
* & * & -\lambda_{1} I & 0 & 0 \\
* & * & * & -\lambda_{2} I & 0 \\
* & * & * & * & \gamma I-R
\end{array}\right] .
$$

Using of Schur complement formula again to (29) yields $\Psi<0$. Therefore, it can be claimed that both $J_{0}<0$ and $V(\tau)-J(\tau, \gamma)<0$ can be guaranteed under the condition (29). It implies that $J_{2} \leq X^{T}(0) P_{1} X(0)$ and $J(\tau, \gamma)>0$. This completes the proof.

Remark 2. The dissipative performance is an extension of robust $H_{\infty}$ and passivity. The passive design methodologies make use of the information of phase, and the $H_{\infty}$ performances aim at the gain of system. The dissipative performance splits the difference between the phase and system gain, and also has less conservatism [39]. When $Q=0, S=I$ and $R=0$ establish, the proposed robust dissipative performance is equal to passivity. Similarly, the proposed dissipative performance is equal to robust $H_{\infty}$ performance when $Q=-I$, $S=0$ and $R=2 \gamma I$. Different from mixed $H_{2} / H_{\infty}$ performance index in $[15,16]$, Theorem 1 provides an anti-disturbance fault tolerant alignment method based on the mixed dissipative/guaranteed cost performance index. This result involves a tuning parameter $\alpha$ and slack variable $P_{2}$, and can lead to less conservative solutions based on the augmented Lyapunov functional approach.

\section{Simulation Examples}

The simulation results are based on experimental data in a laboratory (see Figure 1). The testing conditions are as follows 
- Local latitude is $39.9 \operatorname{deg} N$;

- Initial misalignment angles $\phi_{D}, \phi_{E}, \phi_{N}$ are chosen as 5, 0.5 and 0.5 deg, respectively;

- Gyroscopes: the relative times of Markov process are $\tau_{1}=\tau_{2}=\tau_{3}=3600 \mathrm{~s}$ and random drift is $0.05 \mathrm{deg} / \mathrm{hr}$;

- Accelerometers: the relative times of Markov process are $\tau_{4}=\tau_{5}=1800 \mathrm{~s}$ and random bias is $50 \mu \mathrm{g}$.

Based on the properties of selected sensors, the correlated times of Markov process are determined by the testing method. The coefficient matrices in the nonlinear model (14) are given by

$$
\begin{aligned}
& A=10^{-4} \times\left[\begin{array}{ccc}
0 & -0.467752 & 0 \\
0.467752 & 0 & 0.559426 \\
0 & -0.559426 & 0
\end{array}\right] \\
& D=\left[\begin{array}{lllll}
0 & 0 & 0 & 1 & 0 \\
0 & 0 & 0 & 0 & 1 \\
1 & 0 & 0 & 0 & 0
\end{array}\right], C=\left[\begin{array}{ccc}
0 & 9.801609 & 0 \\
-9.801609 & 0 & 0 \\
0.000047 & 0 & 0.000056
\end{array}\right] \\
& D_{1}=\left[\begin{array}{lll}
10^{-5} g & 10^{-5} g & \frac{0.1 \pi}{648000}
\end{array}\right]^{T}, \\
& E=\left[\frac{0.0001 \pi}{648000}, \frac{0.0001 \pi}{648000}, \frac{0.0001 \pi}{648000}, 10^{-7} g, 10^{-7} g\right]^{T}, \\
& B=\left[\begin{array}{ll}
I_{3} & 0_{3 \times 2}
\end{array}\right], B_{1}=\left[\frac{0.01 \pi}{648000}, \frac{0.01 \pi}{648000}, \frac{0.1 \pi}{648000}\right]^{T}, \\
& G=0.559426 \times 10^{-4} \operatorname{diag}\{1,1,-1\}, \\
& H=0.559426 \times 10^{-4} \operatorname{diag}\{0,0,1\} .
\end{aligned}
$$

Due to the changes of working temperature, environment and aging of integrated circuit, there may exist unpredictable poor performance or faults in the concerned SINS. To verify the performance of the proposed fault tolerant alignment method for the SINS with multiple disturbances and unknown bias fault, a gyroscope bias fault and accelerometer bias faults are considered as shown in Table 1 [34].

Similar to [34], we insert bias faults of inertial sensors into the data obtained from an experiment. Select the parameter matrices as $Q=-I, S=0, R=2 \gamma I$. The dissipative reference output weighting matrices are selected as

$$
\bar{C}_{d}=\left[\begin{array}{llllllll}
0.1 & 0.1 & 0.01 & 0 & 0 & 0 & 0 & 0
\end{array}\right]
$$


Table 1: Fault Type of Inertial Sensor

\begin{tabular}{c|c|c}
\hline Occurrence Position & Magnitude of Fault & Occurrence Time \\
\hline Gyroscope z-axis & $1 \mathrm{deg} / \mathrm{hr}$ & $150 \mathrm{~s}-300 \mathrm{~s}$ \\
Accelerometer x-axis & $3 \mathrm{mg}$ & $150 \mathrm{~s}-300 \mathrm{~s}$ \\
Accelerometer y-axis & $10 \mathrm{mg}$ & $150 \mathrm{~s}-300 \mathrm{~s}$ \\
Accelerometer z-axis & $5 \mathrm{mg}$ & $150 \mathrm{~s}-300 \mathrm{~s}$ \\
\hline
\end{tabular}

The guarantee cost reference output weighting matrices are selected as

$$
\bar{C}_{2}=\left[\begin{array}{llllllll}
10^{-6} g & 10^{-6} g & 10^{-5} g & \frac{0.01 \pi}{180} & \frac{0.01 \pi}{180} & \frac{0.01 \pi}{180} & \frac{0.1 \pi}{180} & \frac{0.1 \pi}{180}
\end{array}\right]
$$

For weighting parameters $\lambda_{1}=0.013211, \lambda_{2}=0.013211$, and tuning parameters $\alpha=20$, it can be solved via LMI related to $(29)$ that $\gamma=2.179985 \times 10^{-11}$, and the gain of multiobjective filter (21) is

$$
\bar{L}=\left[\begin{array}{ccc}
-0.036805 & 0.043127 & 0.999999 \\
-0.026919 & 0.013858 & 0.000000 \\
0.054935 & -0.067048 & 0.000000 \\
-0.000001 & -0.000001 & 0.000000 \\
-0.000005 & 0.000002 & 0.000000 \\
-0.000001 & -0.000000 & 0.000000 \\
-0.001230 & -0.000861 & 0.000000 \\
-0.001230 & -0.000861 & 0.000000 \\
0.141475 & -0.173774 & 0.056144
\end{array}\right]
$$

The parameter matrices of UKF are selected as follows. The initial mean square error matrix is

$$
\begin{aligned}
P(0)= & \operatorname{diag}\left\{(1 \mathrm{deg})^{2},(1 \mathrm{deg})^{2},(10 \mathrm{deg})^{2},(0.2 \mathrm{deg} / \mathrm{hr})^{2},\right. \\
& \left.(0.2 \mathrm{deg} / \mathrm{hr})^{2},(0.2 \mathrm{deg} / \mathrm{hr})^{2},(200 \mu g)^{2},(200 \mu g)^{2}\right\}
\end{aligned}
$$

The variance matrix of system noise is

$$
Q=\operatorname{diag}\left\{(0.1 \mathrm{deg} / \mathrm{hr})^{2},(0.1 \mathrm{deg} / \mathrm{hr})^{2},(0.1 \mathrm{deg} / \mathrm{hr})^{2}\right\},
$$

and variance matrix of measurement noise is selected as

$$
R=\operatorname{diag}\left\{(50 \mu g)^{2},(50 \mu g)^{2},(0.05 \mathrm{deg} / h)^{2}\right\} .
$$

The estimation errors of three misalignment angles are described in Figures 2-5, where the solid lines represent the estimation errors by the proposed anti-disturbance fault tolerant (ADFT) filter in this paper and dash-dot lines denote the estimations errors based on UKF. Figure 2 shows the results with a gyroscope bias fault and Figures 3-5 demonstrate the results with accelerometer bias faults. The standard deviations (STD) of estimation error of 
Table 2: The Standard Deviation (STD) of estimation error with gyroscope z-axis bias fault $\left(10^{-5} \mathrm{rad}\right)$

\begin{tabular}{c|c|c|c}
\hline Method & leveling x & leveling y & azimuth \\
\hline ADFT Filter & 2.04567 & 2.11038 & 5.77020 \\
UKF & 2.87675 & 2.62836 & 29.2468 \\
\hline
\end{tabular}

Table 3: The STD of estimation error with accelerometer x-axis bias fault: $3 \mathrm{mg}\left(10^{-5} \mathrm{rad}\right)$

\begin{tabular}{c|c|c|c}
\hline Method & leveling x & leveling y & azimuth \\
\hline ADFT Filter & 6.28524 & 2.29582 & 13.8386 \\
UKF & 16.3337 & 3.40254 & 28.3640 \\
\hline
\end{tabular}

three misalignment angles (after the 30th second) between the proposed approach and UKF are shown in Tables II-V. From Figures 2-5 and Tables II-V, it can be seen that the proposed ADFT filter has a good ability for initial alignment of SINS with multiple disturbances and sensor faults, and the UKF is degraded. It is shown that the proposed filter has enhanced disturbance rejection and attenuation performance, and high reliability. Because of the poor observable degree of azimuth misalignment angle, the estimation error of azimuth is less accuracy than level misalignment angles.

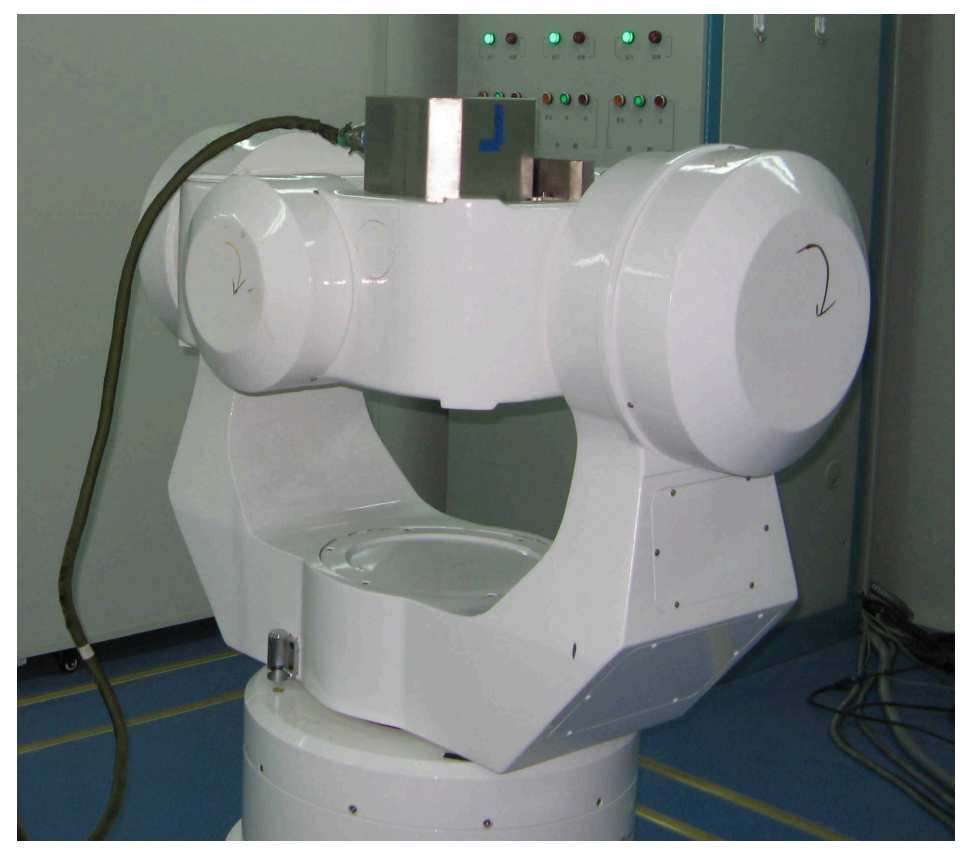

Figure 1: Triaxial test turntable

\section{Conclusion}

In this paper, the problem of initial alignment is investigated for INS with multiple disturbances and sensor faults. There are the following features of the proposed algorithm 

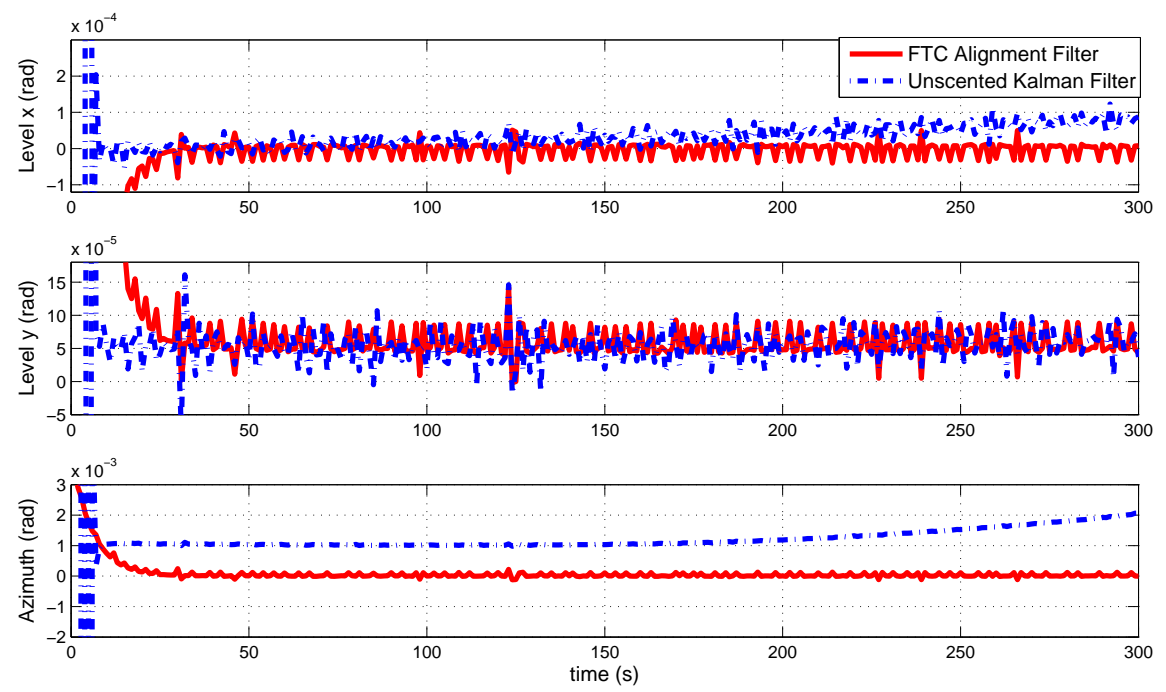

Figure 2: Gyroscope z-axis bias fault: $1 \mathrm{deg} / \mathrm{hr}$
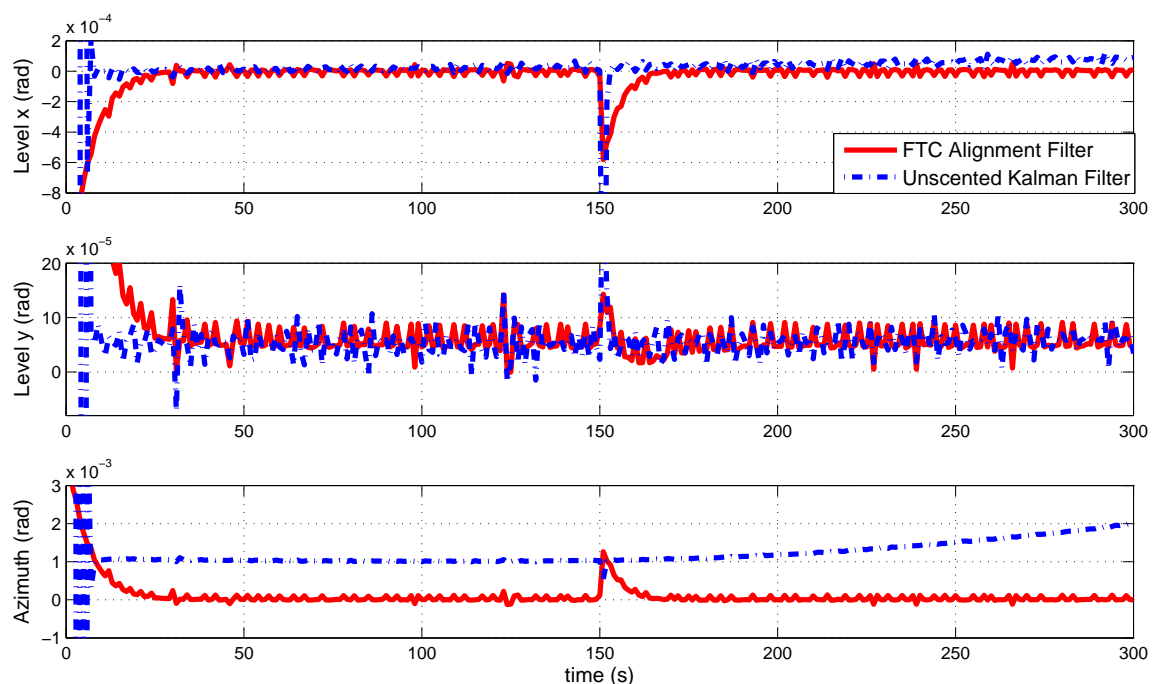

Figure 3: Accelerometer $\mathrm{x}$-axis bias fault: $3 \mathrm{mg}$

Table 4: The STD of estimation error with accelerometer y-axis bias fault: $10 \mathrm{mg}\left(10^{-5} \mathrm{rad}\right)$

\begin{tabular}{c|c|c|c}
\hline Method & leveling x & leveling y & azimuth \\
\hline ADFT Filter & 3.51955 & 14.7711 & 8.80387 \\
UKF & 8.21254 & 53.8093 & 29.6776 \\
\hline
\end{tabular}



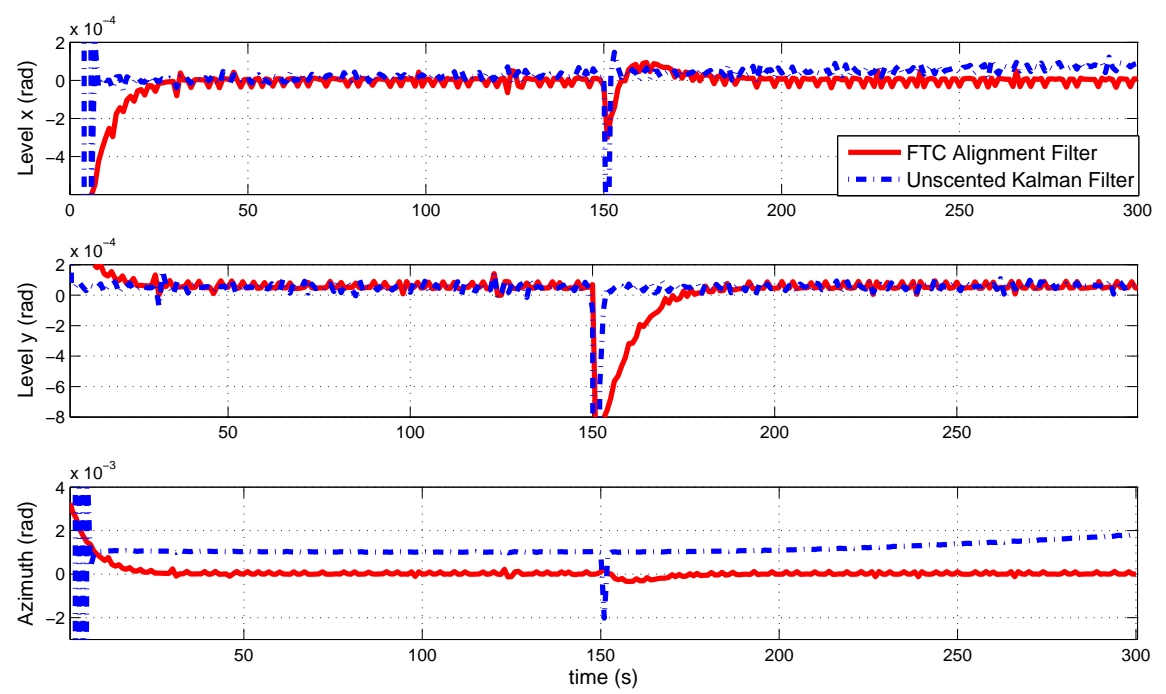

Figure 4: Accelerometer y-axis bias fault: $10 \mathrm{mg}$
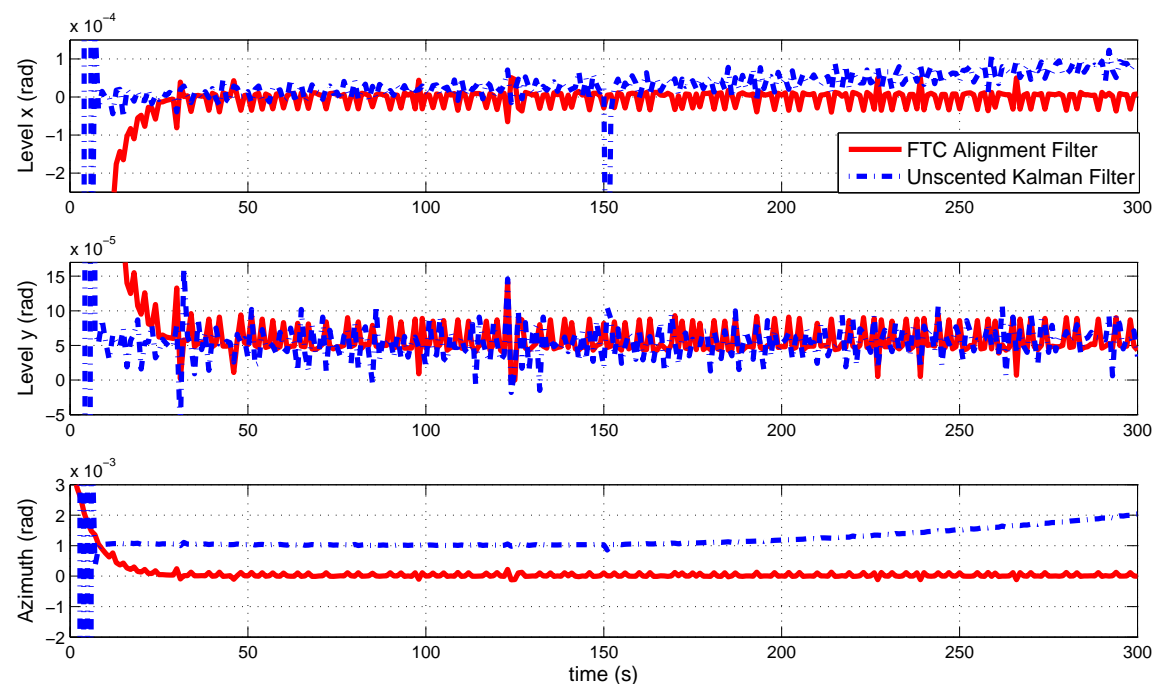

Figure 5: Accelerometer z-axis bias fault: $5 \mathrm{mg}$

Table 5: The STD of estimation error with accelerometer z-axis bias fault: $5 \mathrm{mg}\left(10^{-5} \mathrm{rad}\right)$

\begin{tabular}{cccc}
\hline Method & leveling x & leveling y & azimuth \\
\hline ADFT Filter & 2.04612 & 2.11129 & 5.77145 \\
UKF & 6.06999 & 2.64613 & 28.7699 \\
\hline
\end{tabular}


compared with the previous results.

- Firstly, a new SINS model with multiple disturbances and sensor faults is established instead of lumping all the errors and disturbances together as a Gaussian noise or bounded noise. In our approach, inertial sensor error is represented as a first order Markov process, while modeling error, environmental disturbance and measuremen$\mathrm{t}$ noise are merged into a norm bounded disturbance. Then, the initial alignment problem is transformed into a filter design problem of nonlinear systems with multiple disturbances and faults.

- Secondly, an anti-disturbance fault tolerant filter is designed for the concerned nonlinear systems with both disturbance rejection and attenuation performance. For systems with multiple disturbances and faults, classical Kalman filtering type methods may provide a degraded performance and accuracy. By taking advantage of disturbance characteristics, corresponding compensation terms are applied to reject inertial sensor error and accommodate sensor fault. Robust multi-objective performance is used for optimizing the system performance.

- Thirdly, introducing slack variables and dissipative performance can reduce conservatism of the proposed approach. Comparison results show that the proposed method has higher accuracy and reliability.

\section{References}

[1] J. A. Farrell, M. Barth, The Global Positioning System \& Inertial Navigation, McGraw -Hall companies Inc, USA, 1999.

[2] R. M. Rogers, Applied Mathematics in Integrated Navigation Systems, 3rd Edition, AIAA Inc, Virginia, USA, 2007.

[3] A. Acharya, S. Sadhu, T. K. Ghoshal, Improved self-alignment scheme for SINS using augmented measurement, Aerospace Science and Technology. 15 (2) (2011) 125-128.

[4] H. S. Ahn, C. H. Won, Fast alignment using rotation vector and adaptive kalman filter, IEEE Trans. Aero. Elec. Sys 42 (1) (2006) 70-83.

[5] X. Wang, X. Guan, J. Fang, H. Li, A high accuracy multiplex two-position alignment method based on sins with the aid of star sensor, Aerospace Science and Technology 42 (2015) 66-73.

[6] J. C. Fang, S. Yang, Study on innovation adaptive EKF for in-flight alignment of airborne POS, IEEE Trans. Instrum. Meas. 60 (4) (2011) 1378-1388.

[7] E. H. Shin, N. El-Sheimy, An unscented kalman filter for in-motion alignment of low-cost IMUs, Position Location and Navigation Symposium of IEEE, 2004, pp. 273-279.

[8] L. Xie, C. E. De Souza, Y. Wang, Robust filtering for a class of discrete-time uncertain nonlinear systems: An $H_{\infty}$ approach, International Journal of Robust and Nonlinear Control 6 (6) (2015) 297312.

[9] Z. Wang, H. Dong, B. Shen, H. Gao, Finite-horizon $H_{\infty}$ filtering with missing measurements and quantization effects, IEEE Transactions on Automatic Control 58 (7) (2013) 1707-1718.

[10] J. Ali, M. Ushaq, A consistent and robust kalman filter design for in-motion alignment of inertial navigation system, Measurement 42 (2) (2009) 577-582.

[11] M. J. Yu, J. G. Lee, C. G. Park, Nonlinear robust observer design for strapdown INS in-flight alignment, IEEE Trans. Aero. Elec. Sys 40 (3) (2004) 797-807.

[12] Z. Gao, Y. Zhong, D. Mu, S. Gao, Robust adaptive filter allowing systematic model errors for transfer alignment, Aerospace Science and Technology 59 (2016) 32-40. 
[13] L. Guo, S. Y. Cao, Anti-Disturbance Control for Systems with Multiple Disturbances, CRC Press, USA, Boca Raton, 2013.

[14] S. Li, J. Yang, W. Chen, X. Chen, Disturbance Observer-based Control: Methods and Applications, CRC Press, Boca Raton, 2014.

[15] S. Y. Cao, L. Guo, Multi-objective robust initial alignment algorithm for inertial navigation system with multiple disturbances, Aerosp. Sci. Technol 21 (1) (2012) 1-6.

[16] L. Guo, S. Y. Cao, Initial alignment for nonlinear inertial navigation systems with multiple disturbances based on enhanced anti-disturbance filtering, Int. J. Control 85 (5) (2012) 491-501.

[17] J. J. Gertler, Fault Detection and Diagnosis in Engineering Systems, Marcel Dekker, New York, 1998.

[18] J. Lan, R. J. Patton, A new strategy for integration of fault estimation within fault-tolerant control, Automatica 69 (2016) 48-59.

[19] Y. M. Zhang, J. Jiang, Bibliographical review on reconfigurable fault-tolerant control systems, Annu. Rev. Control 32 (2008) 229-252.

[20] Z. Gao, C. Cecati, S. X. Ding, A survey of fault diagnosis and fault-tolerant techniquesPart I: Fault diagnosis with model-based and signal-based approaches, IEEE Trans Industrial Electronics 62 (6) (2015) 3757-3767.

[21] D. H. Zhou, X. He, Z. D. Wang, G. P. Liu, Y. D. Ji, Leakage fault diagnosis for an internet-based three-tank system: an experimental study, IEEE Trans Contr Syst Technol. 20 (4) (2012) 857-870.

[22] K. Zhang, B. Jiang, P. Shi, J. Xu, Multi-constrained fault estimation observer design with finite frequency specifications for continuous-time systems, International Journal of Control 87 (8) (2014) 1635-1645.

[23] S. X. Ding, B. Shen, Z. Wang, M. Zhong, A fault detection scheme for linear discrete-time systems with an integrated online performance evaluation, International Journal of Control 87 (12) (2014) 2511-2521.

[24] X. D. Zhang, M. M. Polycarpou, T. Parisini, Fault diagnosis of a class of nonlinear uncertain systems with lipschitz nonlinearities using adaptive estimation, Automatica 46 (2010) 290-299.

[25] Q. Hu, X. Shao, Smooth finite-time fault-tolerant attitude tracking control for rigid spacecraft, Aerospace Science and Technology 55 (2016) 144-157.

[26] B. Xiao, S. Yin, O. Kaynak, Tracking control of robotic manipulators with uncertain kinematics and dynamics, IEEE Transactions on Industrial Electronics 63 (10) (2016) 6439-6449.

[27] B. Xiao, S. Yin, L. Wu, A structure simple controller for satellite attitude tracking maneuver, IEEE Transactions on Industrial Electronics 64 (2) (2017) 1436-1446.

[28] B. Xiao, S. Yin, Velocity-free fault-tolerant and uncertainty attenuation control for a class of nonlinear systems, IEEE Transactions on Industrial Electronics 63 (7) (2016) 4400-4411.

[29] J. C. Fang, J. L. Li, Integrated model and compensation of thermal errors of silicon microelectromechanical gyroscope, IEEE Trans. Instrum. Meas. 58 (9) (2009) 2923-2930.

[30] M. El-Diasty, A. El-Rabbany, S. Pagiatakis, Temperature variation effects on stochastic characteristics for low-cost MEMS-based inertial sensor error, Meas. Sci. Technol. 18 (11) (2007) 3321.

[31] U. I. Bhatti, W. Y. Ochieng, S. Feng, Integrity of an integrated GPS/INS system in the presence of slowly growing errors. Part I: A critical review, GPS Solut. 11 (3) (2007) 173-181.

[32] S. Sukkarieh, E. M. Nebot, H. F. Durrant-Whyte, A high integrity IMU/GPS navigation loop for autonomous land vehicle applications, IEEE Trans. Robotics Autom. 15 (3) (1999) 572-578.

[33] R. Wang, Z. Xiong, J. Liu, J. Xu, Chi-square and sprt combined fault detection for multisensor navigation, IEEE Transactions on Aerospace and Electronic Systems 52 (3) (2016) 1352-1365.

[34] K. H. Kim, J. G. Lee, Adaptive two-stage EKF for a fault tolerant INS-GPS loosely coupled system, IEEE Trans. Aero. Elec. Sys. 45 (1) (2009) 125-137.

[35] S. G. Park, H. C. Jeong, J. W. Kim, D.-H. Hwang, S. J. Lee, Magnetic compass fault detection method for GPS/INS/magnetic compass integrated navigation systems, International Journal of Control, Automation and Systems 9 (2) (2011) 276-284.

[36] Y. Q. Liang, Y. M. Jia, A nonlinear quaternion-based fault-tolerant SINS/GNSS integrated navigation method for autonomous UAVs, Aerosp. Sci. Technol. 40 (2015) 191-199.

[37] B. Scherzinger, Inertial navigator error models for large heading uncertainty, Proc. IEEE Position Location and Navigation Symposium, Atlanta, USA, 1996, pp. 477-484. 
[38] E. Nebot, H. Durrant-Whyte, Initial calibration and alignment of low cost inertial navigation units for land vehicle applications, J. Robot. System 16 (2) (1999) 81-92.

[39] B. Brogliato, R. Lozano, B. Maschke, O. Egeland, Dissipative Systems Analysis and Control: Theory and Applications, 2nd Edition, Springer Verlag, London, 2007. 